Author receives speaker honoraria for Edwards Lifesciences.

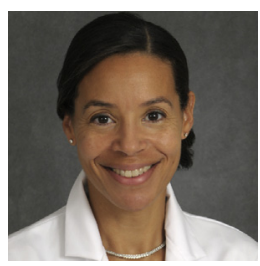

\section{THE BIOPROSTHETIC VERSUS MECHANICAL VALVE DEBATE: UNWINNABLE AND INCREASINGLY IRRELEVANT? \\ Reply to the Editor:}

It is a remarkable fact that surgeons and specialty leaders, including 3 American Association for Thoracic Surgery presidents, are divided between 2 therapies as different as mechanical and bioprosthetic valve replacement - split between stances that range from advocating mechanical valves in the elderly to recommending bioprosthetic valves in the young. ${ }^{1}$

It is remarkable, but it is not surprising. The enormous pile of relevant evidence can be selectively quoted or critiqued to support both positions and the entire spectrum in between. Dr Emiliano Rodriguez-Caulo ${ }^{2}$ outlines some of the issues in his critique of the meta-analysis of mechanical versus bioprosthetic valve published recently in the Journal, ${ }^{3}$ and he then goes on to describe ANDAVALVE, a prospective, observational registry trial-but even this methodology cannot adequately address the selection bias that undermines the reliability of every nonrandomized study.

Is a randomized trial the answer? Only if we can wait 20 years for survival data that would inform practice, but obviously that is too long in a specialty where patient preference and rapid advances in technology are the primary drivers of practice change, and where it took less than a decade for transcatheter valve replacement to go from first-in-human to the dominant therapy for valve replacement in aortic stenosis.

The Achilles' heel of transcatheter technology is calcific degeneration of xenografts. Solving this could establish transcatheter aortic valve surgery as the first choice for patients in all age groups. The solution looks increasingly likely to lie in emerging polymer technology, which in initial hemodynamic in vitro testing has shown improved hemodynamics, minimization of platelet activation and thrombosis, and improved strength, mechanical stability, and durability. Recent work suggests that a novel polymeric transcatheter valve outperforms clinically used xenograft transcatheter valves in vitro, and it hints at the possibility of a future new clinical pradigm. ${ }^{4}$

In the interim, producing more nonrandomized data comparing mechanical and bioprosthetic valves just adds to the mounting slush pile of evidence without materially informing or shifting the debate. Knowledge gaps that we could usefully tackle instead include the incidence of structural valve degeneration at 5 years after transcatheter valve replacement in patients at intermediate and low risk, strategies to minimize the operative mortality of reoperative aortic valve replacement and optimize valve-in-valve outcomes, and the role of novel anticoagulants in reducing bioprosthetic valve thrombosis and structural degeneration.

Wherever we choose to focus, it is time to stop repeating studies with the same methodologic problems. This is an unwinnable debate. And it is one that may soon be irrelevant.

Joanna Chikwe, MD, FRCS

Department of Cardiovascular Surgery Mount Sinai Medical Center

New York, NY

Department of Surgery

The State University of New York

Stony Brook, NY

\section{References}

1. Sundt T, Schaff H, Soltesz EG, Uva MS, Adams DH. Mechanical vs biologic valves: our modern day conundrum. Semin Thorac Cardiovasc Surg. 2016;28: 404-17.

2. Rodriguez-Caulo E. More evidence favoring bioprostheses for patients older than 55 years in aortic valve replacement. J Thorac Cardiovasc Surg. 2019;157:e357. 3. Diaz R, Hernandez-Vaquero D, Alvarez-Cabo R, Avanzas P, Silva J, Moris C, et al. Long-term outcomes of mechanical versus biological aortic prosthesis: systematic review and meta-analysis. J Thorac Cardiovasc Surg. November 20, 2018 [Epub ahead of print].

4. Rotmen OM, Kovarovic B, Chiu W, Bianchi M, Marom G, Slepian MJ, et al. Novel polymeric valve for transcatheter aortic valve replacement applications: in vitro hemodynamic study. Ann Biomed Eng. 2019;47:113-25.

https://doi.org/10.1016/j.jtcvs.2019.02.086

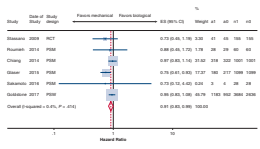

\section{SHOULD WE MODIFY THE PROTOCOL OF A SYSTEMATIC REVIEW TO INCLUDE A RELEVANT STUDY? Reply to the Editor:}

We thank Rodriguez-Caulo ${ }^{1}$ for his letter and Chikwe and Weiss ${ }^{2}$ for their editorial commentary on our systematic review and meta-analysis on mechanical versus biological valves. We concluded that, compared with biological valves, mechanical aortic prostheses provide better long-term survival.

Under ideal conditions, the best evidence comes from the meta-analysis of randomized, controlled trials (RCTs). This is not the case for this issue, however, because there is only a single RCT with small statistical power. Overcoming this limitation is the main goal of any meta-analysis. Propensity score methods are used to deal with the intrinsic biases of observational studies. In addition, propensity score matching allows direct comparison of survival curves. ${ }^{3}$ So 


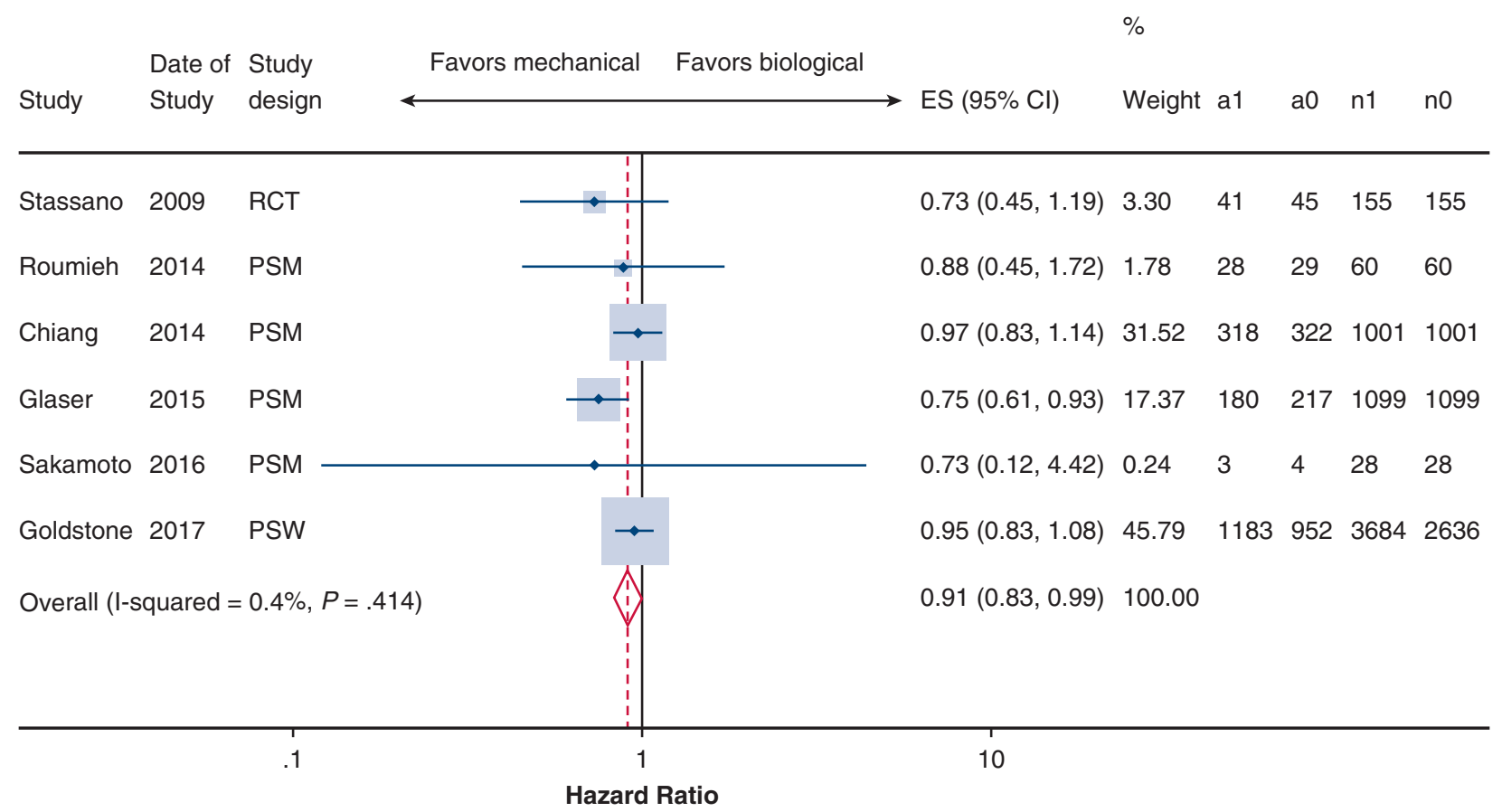

A

\begin{tabular}{|c|c|c|c|c|c|c|c|c|}
\hline \multirow{3}{*}{ Study } & \multirow{3}{*}{\multicolumn{2}{|c|}{$\begin{array}{ll}\text { Date of } & \text { Study } \\
\text { Study } & \text { design }\end{array}$}} & & & \multicolumn{4}{|c|}{$\%$} \\
\hline & & & & & ES $(95 \% \mathrm{Cl})$ & Weight a1 & $\mathrm{a} 0$ & $\mathrm{n} 1$ \\
\hline & & & Favors mechanical & Favors biological & & & & \\
\hline
\end{tabular}

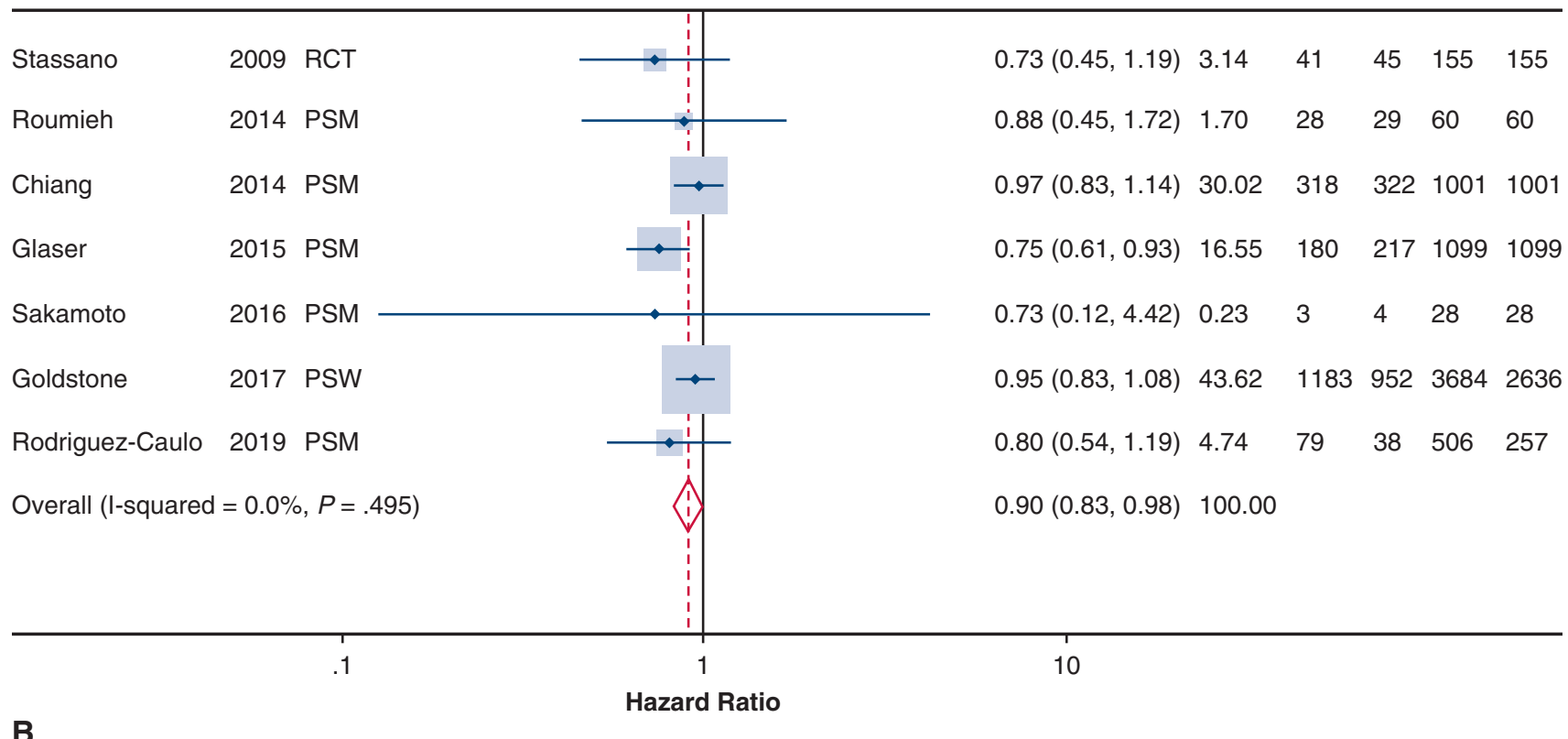

FIGURE 1. A, Forest plot for late survival if we had included the study by Goldstone and associates. ${ }^{5}$ The lower hazard ratio for the mechanical valve group versus the biological valve group indicates better survival in the mechanical valve group. The statistic $\mathrm{I}^{2}=0.4 \%$ indicates the near absence of inconsistency or heterogeneity. B, Forest plot for late survival if we had included both the study by Goldstone and associates ${ }^{5}$ and the study by Rodriguez-Caulo and colleagues. ${ }^{4}$ The lower hazard ratio for the mechanical valve group versus the biological valve group indicates better survival in the mechanical valve group. The statistic $\mathrm{I}^{2}=0 \%$ indicates an absence of inconsistency or heterogeneity. ES, Estimation (hazard ratio); $C I$, confidence interval; RCT, randomized, controlled trial; PSM, propensity score matched; PSW, propensity score weighted. 
Authors have nothing to disclose with regard to commercial support.

propensity score matching, as Rodriguez-Caulo and colleagues ${ }^{4}$ and Chikwe ${ }^{5}$ used in their good works, is a good alternative when RCTs are not possible or are hard to achieve.

In November 2017, the largest study on this issue was published in The New England Journal of Medicine by Goldstone and associates. ${ }^{6}$ Unfortunately, we could not include it, because it did not meet one of the inclusion criteria of our previously published protocol. It was not a propensity score-matching study, but rather a propensity score-weighted study. The only solution would have been to change the inclusion criteria with the sole objective of including this study. We considered it very carefully.

To prevent biases, we decided that introduction, conclusions, journal identification, and other details should not be available during the selection process. Should we modify our protocol to include a study published in the prestigious New England Journal of Medicine? Clearly, our meta-analysis would be improved with very little added effort. Would this, however, be ethical? Does the noninclusion of an observational and retrospective study that is based on a registry invalidate our meta-analysis just because it is large, well-designed, and published in The New England Journal of Medicine? Ultimately, we decided not to modify the protocol.

Let us imagine, however, that we had changed the protocol so that we could include the study of Goldstone and associates. ${ }^{6}$ Figure 1, A, shows the result. The conclusion would be similar! Let us think now that we could have included the even newer published article by
Rodriguez-Caulo and colleagues ${ }^{4}$ (Figure 1, B). The conclusion would be similar again! There is great consistency among all the studies.

Therefore, until more RCTs have been published, the available evidence will not generate blind beliefs. Until then, we can trust this meta-analysis, knowing that even the moon has spots and still is great.

Daniel Hernandez-Vaquero, $M D, P h D^{a, b}$
Rocio Diaz, $M D, P h D^{a, b}$
Cesar Moris, $M D, P h D^{a, b, c}$
${ }^{a}$ Heart Area
Hospital Universitario Central de Asturias
Oviedo, Spain
${ }^{b}$ Instituto de Investigación Sanitaria del Principado de
Asturias
Oviedo, Spain
${ }^{c}$ University of Oviedo
Oviedo, Spain

\section{References}

1. Rodriguez-Caulo EA. More evidence favoring bioprosthesis older than 55 years in aortic valve replacement. J Thorac Cardiovasc Surg. 2019;157:e357.

2. Chikwe J, Weiss A. The mid-life crisis of the meta-analysis. J Thorac Cardiovasc Surg. November 27, 2018 [Epub ahead of print].

3. Deb S, Austin PC, Tu JV, Ko DT, Mazer CD, Kiss A, et al. A review of propensity-score methods and their use in cardiovascular research. Can J Cardiol. 2016;32:259-65.

4. Rodríguez-Caulo EA, Macías D, Adsuar A, Ferreiro A, Arias-Dachary J, Parody G, et al. Biological or mechanical prostheses for isolated aortic valve replacement in patients aged 50-65 years: the ANDALVALVE study. Eur $J$ Cardiothorac Surg. January 3, 2019 [Epub ahead of print].

5. Chiang YP, Chikwe J, Moskowitz AJ, Itagaki S, Adams DH, Egorova NN Survival and long term outcomes following bioprosthetic vs mechanical aortic valve replacement in patients aged 50 to 69 years. JAMA. 2014:312:1323-9.

6. Goldstone AB, Chiu P, Baiocchi M, Lingala B, Patrick WL, Fischbein MP, et al Mechanical or biologic prostheses for aortic-valve and mitral-valve replacement. N Engl J Med. 2017;377:1847-57.

https://doi.org/10.1016/j.jtcvs.2019.02.036 